\title{
Influência da Condição de Emprego/Desemprego dos Pais na Exploração e Indecisão Vocacional dos Adolescentes
}

\author{
Influence of Parents' Employment/Unemployment Condition in Adolescents' \\ Career Exploration and Indecision
}

\author{
Liliana Costa Faria* \\ Universidade Europeia, Lisboa, Distrito de Lisboa, Portugal
}

\begin{abstract}
Resumo
Este estudo centra-se no impacto da condição de emprego/desemprego dos pais nas crenças, comportamentos e reações de exploração vocacional e na indecisão de carreira dos adolescentes. Foram administradas o Career Exploration Survey e o Career Decision Scale a uma amostra de 321 adolescentes Portugueses, de ambos os sexos (190, 59,2\% raparigas; 131, 40,8\% rapazes), com idades compreendidas entre os 13 e os 17 anos. Os resultados indicam que os adolescentes filhos de pais desempregados manifestam, significativamente, crenças mais negativas acerca do mercado de trabalho, menos comportamentos exploratórios, e reações afetivas mais negativas do que os adolescentes filhos de pais empregados e, consequentemente, níveis mais elevados de indecisão vocacional. Os resultados são discutidos, retirando-se implicações para a intervenção psicológica e salientando-se direcções para futuras investigações.

Palavras-chave: Orientação vocacional, papel dos pais, influência parental, escolha de carreira, indecisão vocacional.
\end{abstract}

\begin{abstract}
This study focuses on the impact of parents' employment/unemployment condition on the beliefs, behaviors and reactions of career exploration and indecision of adolescents. Career Exploration Survey and Career Decision Scale were administered to a sample of 321 Portuguese adolescents of both sexes (190 women - 59.2\% and 131 men - 40.8\%) aged between 13 and 17 years. The results indicate that the adolescents whose parents are unemployed express significantly less positive beliefs about the labor market, less exploratory behaviors, and less positive emotional reactions than the adolescents whose parents are employed and, consequently, higher levels of career indecision. The results are discussed based on the implications of psychological intervention and point out directions for future research.

Keywords: Career guidance, parental role, parental influence, career choice, career indecision.
\end{abstract}

O tema da influência dos pais no desenvolvimento vocacional dos adolescentes tem sido um dos aspetos mais recentemente valorizados, quer dentro (e.g., Carvalho \& Taveira, 2009, 2010; Murray, 2009; Pratta \& Santos, 2007), como fora (e.g., Alarcão \& Gaspar, 2007; Boing, Crepaldi, \& More, 2008) da literatura vocacional, sobretudo no que diz respeito aos anos do ensino básico e secundário. Os diversos modelos conceituais, nomeadamente os desenvolvimentistas, contextualistas e construtivistas, defendem que o desenvolvimento vocacional é um processo contínuo, que se constrói desde a infância até à velhice, sendo resultado da interação entre a pessoa e os múltiplos contextos de vida em que ela se insere. Entre os vários contextos, a

"Endereço para correspondência: Universidade Europeia, Laureate International Universities, Estrada da Correia, 53, Campus Lisboa, Lisboa, Distrito de Lisboa, Portugal 1500-210. E-mail: liliana.faria@europeia.pt família constitui-se como o primeiro, e o mais significativo, para os adolescentes (Gonçalves \& Coimbra, 2007; Otto, 2000), apoiando-os na concretização das diversas tarefas vocacionais, tais como a construção de uma identidade, a aquisição de uma perspetiva temporal, a formulação de preferências vocacionais ou o desenvolvimento de consciência face à necessidade de tomada de decisão (e.g., Carvalho \& Taveira, 2010; Diemer, 2007; Murray, 2009).

Do mesmo modo, as inúmeras investigações que têm sido realizadas, neste âmbito, (e.g., Carvalho \& Taveira, 2010; Creamer \& Laughlin, 2005; Gonçalves, 2008; Otto, 2000; Pinto \& Soares, 2002; Pratta \& Santos, 2007; Whiston \& Keller, 2004; Young et al., 2006) parecem, igualmente, unânimes em reconhecer a importância dos pais no desenvolvimento vocacional dos adolescentes. Tais investigações resultaram num conjunto substancial de variáveis associadas à família e, consideradas como capazes de influenciar a escolha, o compromisso, e a execução dos planos de carreira dos adolescentes. Uma dessas variáveis 
é a condição de emprego versus desemprego dos pais (e.g., Gonçalves, 2008; Hartung, Porfeli, \& Vondracek, 2005). Estudos realizados, neste âmbito (Bacon \& Lerner, 1975; Castellino, Lerner, Lerner, \& Eye, 1999; Cinamon, 2002; Neblett \& Cortina, 2006; Sobral, Gonçalves, \& Coimbra, 2009), apontam o desemprego como uma das situações profissionais parentais que pode influenciar a escolha vocacional dos filhos.

Para Leon e Iguti (2003), o desemprego enquanto fenómeno mundial, e de grande incidência na contemporaneidade, representa privações e ruturas nas mais variadas esferas da vida do ser humano, sendo que o seu impacto estende-se não apenas ao indivíduo desempregado, mas também a todo o seu contexto familiar. Segundo Vasconcelos e Oliveira (2004), o desemprego desequilibra o ambiente familiar, a autoconfiança e, consequentemente, reflete de maneira negativa nos vínculos sócio afetivos. Hanisch (1999) refere que o desemprego afeta a tranquilidade dos pais, a união familiar, provocando aumento dos conflitos, violência ou irritabilidade parental, influenciando, negativamente, a relação com os filhos (Crouter \& Bumpus, 2001). Neste âmbito, o aumento das taxas de desemprego é um fator com impacto relevante, assumido na literatura como uma crise que ocorre no contexto familiar (Sobral et al., 2009) e, que cria uma nova dinâmica familiar, que aumenta a probabilidade de despertar nos filhos sentimentos de confusão, raiva e insegurança que afetam quer as atitudes destes para com os pais, com a escola e, com o mundo do trabalho quer, o seu relacionamento com o meio e, consequentemente, as suas perceções acerca do seu próprio futuro enquanto trabalhadores (Schliebner \& Peregoy, 1994). Desta forma, a construção de projetos vocacionais por parte dos adolescentes será influenciada, pela comunicação, pela qualidade relacional do ambiente familiar, pelos valores, pela quantidade de apoio percebida, pela congruência de expetativas pais-filhos e, pela perceção de reconhecimento e valorização social dos filhos face aos pais (Gonçalves, 2006; Sobral et al., 2009; Vasconcelos \& Oliveira, 2004; Whiston \& Keller, 2004).

Segundo vários autores, o desemprego parental relaciona-se positivamente com pessimismo parental (e.g.,
Galambos \& Silbereisen, 1987; Neblett \& Cortina, 2006). Os pais desempregados tendem a desenvolver uma convicção generalizada de que um futuro positivo e bem-sucedido dificilmente será alcançável, podendo disseminar nos seus filhos uma visão pessimista do seu próprio futuro ocupacional, tendo consequências a nível: (a) das percepções de auto-eficácia na tomada de decisão (e.g., Turner \& Lapan, 2002), (b) dos processos de desenvolvimento de identidade vocacional (e.g., Bardagi \& Hutz, 2008; Chope, 2006), (c) do grau de compromisso vocacional (e.g., Berríos-Allison, 2005; Chope, 2006; Wolfe \& Betz, 2004), (d) do comportamento exploratório (Amundson \& Penner, 1998; Galambos \& Silbereisen, 1987; Ketterson \& Blustein, 1997; Kracke, 1997; Schliebner \& Peregoy, 1994; Vignolli, Croity-Belz, Chapeland, Fillipis, \& Garcia, 2005) e, (e) da dificuldade de tomada de decisões (Lindstrom, Doren, Metheny, Johnson, \& Zane, 2007; Neblett \& Cortina, 2006).

Nesta linha de investigação, com este estudo pretende investigar-se como a condição de emprego/desemprego dos pais poderá influenciar a construção de projectos vocacionais dos jovens e adolescentes, ao nível das suas crenças, comportamentos e reações afetivas de exploração vocacional, e ao nível da prontidão dos adolescentes face aos processos de tomada de decisão vocacional.

\section{Método}

\section{Participantes}

A amostra desta investigação é constituída por 321 adolescentes portugueses, de ambos os sexos (190, 59,2\% raparigas; $131,40,8 \%$ rapazes), a frequentar o $9^{\circ}$ ano de escolaridade (o $9^{\circ}$ ano do sistema de ensino português corresponde ao último ano do ensino fundamental no sistema educativo brasileiro), em três escolas públicas da região norte de Portugal. A média das idades dos alunos que constituem a amostra é de 14,15 anos $(D P=0,49)$ com idades compreendidas entre os 13 e os 17 anos. No que concerne à condição de emprego/desemprego dos pais, $44,55 \%$ dos alunos identificou apenas um progenitor como estando desempregado e 55,45\% são filhos de pais, ambos, empregados. A amostra é caracterizada na Tabela 1.

Tabela 1

Caracterização da Amostra

\begin{tabular}{lcccc}
\hline Variáveis & & $\begin{array}{c}\text { Pais Desempregados } \\
(n=143)\end{array}$ & $\begin{array}{c}\text { Pais Empregados } \\
(n=178)\end{array}$ & $\begin{array}{c}\text { Total } \\
(N=321)\end{array}$ \\
\hline Idade média $(D P)$ & & $14,20(0,48)$ & $14,12(0,50)$ & $14,15(0,49)$ \\
\multirow{2}{*}{ Sexo } & Raparigas & $70(49,00 \%)$ & $120(67,40 \%)$ & $190(59,20 \%)$ \\
& Rapazes & $73(51,00 \%)$ & $58(32,60 \%)$ & $131(40,80 \%)$
\end{tabular}

\section{Instrumentos de Medida e Procedimentos}

A exploração vocacional foi avaliada através do Career Exploration Survey (CES; Stumpf, Colarelli, \& Hartman,
1983; adap. Taveira, 1997). Na sua versão adaptada é constituída por 54 itens, 53 dos quais em formato likert (escala de cinco categorias de resposta nos itens 1 a 43 e 
sete categorias nos itens 44 a 53), distribuídos por 12 subescalas, organizadas em três dimensões: crenças (estatuto de emprego, certeza nos resultados da exploração, instrumentalidade externa, instrumentalidade interna, importância de obter a posição preferida), comportamentos (exploração com locus no mundo escolar e profissional, exploração com locus em si próprio, exploração sistemática-intencional, quantidade de informação), e reações (satisfação com a informação, stress na exploração, stress na tomada de decisão) relacionadas com a exploração vocacional (Taveira, 1997). O item 54 permite aos participantes indicarem o número de domínios vocacionais explorados. Esta escala revelou qualidades psicométricas satisfatórias em amostras com adolescentes portugueses que frequentavam o $9^{\circ}$ ano de escolaridade $(N=700)$, com uma precisão de medida, obtida pelo alpha de Cronbach, entre 0,63 e 0,86 (Taveira, 1997).

A indecisão vocacional foi avaliada através do Career Decision Scale (CDS; Osipow, Carney, Winer, Yanico, \& Koshier, 1976; adap. por Taveira, 1997). Esta escala permite avaliar o nível de prontidão de adolescentes face aos processos de tomada de decisão vocacional. É constituída por 12 itens, 11 dos quais formulados numa escala de resposta do tipo likert, com quatro categorias, num formato gráfico-numérico. O item 12 , de resposta aberta, possibilita que cada participante apresente informação mais detalhada sobre o seu estado de (in)decisão, caso alguma das situações referidas nos itens restantes não se apliquem. Numa amostra de adolescentes portugueses do $9^{\circ}$ ano, as estimativas de consistência interna, estimadas pelo coeficiente alpha, foram satisfatórias, situando-se entre 0,88 e 0,91 (Taveira, 1997).

Inicialmente foi pedido autorização, às escolas envolvidas, para a recolha de dados e desenvolvimento do estudo, as quais acederam sem quaisquer restrições. Também foram pedidas autorizações aos pais dos alunos envolvidos no estudo.

Os dados relativos à condição de emprego/desemprego dos pais foram recolhidos mediante a formulação de questões num questionário socio-demográfico, aplicado simultaneamente ao CES e CDS.

A aplicação dos instrumentos junto dos alunos foi coletiva e abrangeu todos os adolescentes da amostra numa única sessão. Inicialmente foram apresentados aos alunos os objetivos do estudo e o interesse na aplicação dos instrumentos de pesquisa, bem como prestados outros esclarecimentos, tais como a necessidade de serem verdadeiros nas suas respostas, e a garantia de confidencialidade das respostas dadas. De seguida, foram distribuídos os consentimentos informados, e um caderno com os questionários. O tempo médio de preenchimento dos instrumentos foi de 40 minutos. Não foi feita qualquer submissão a Comitê de Ética, dado não ser um padrão requerido em Portugal.

Foram realizadas análises descritivas e $T$ testes para analisar a existência de diferenças estatisticamente significativas na exploração e indecisão de carreira em função da condição de emprego/desemprego dos pais e do sexo. Os resultados foram considerados estatisticamente significativos quando o valor do teste de significância foi inferior a $0,05(p<0,05)$.

\section{Resultados}

A Tabela 2 apresenta os resultados das análises de estatística descritiva e da análise das diferenças entre as médias da exploração e indecisão de carreira, em função da condição de emprego/desemprego dos pais. Constata-se a existência de diferenças estatisticamente significativas entre filhos de pais em condição de emprego e filhos de pais em que um pai (ou mãe) está na condição de desemprego, no sentido de crenças mais negativas de exploração, menos comportamentos de exploração e, reações afetivas mais negativas à exploração vocacional, para estes últimos (filhos de pais em que um deles está na condição de desemprego).

A análise da Tabela 2 permite verificar a existência de diferenças estatisticamente significativas nos dois grupos (condição de emprego versus desemprego) para as dimensões: Estatuto de Emprego, Exploração com locus no Meio, Exploração com locus em Si Próprio/a, Quantidade de Informação, Satisfação com a Informação e Stress na Tomada de Decisão. Mais especificamente, os filhos de pais desempregados, quando comparados com os filhos de pais empregados, exibem uma visão mais negativa do mercado de trabalho, acreditando menos na probabilidade de existência de emprego na sua área profissional preferida (Estatuto de Emprego), apresentam menor grau de exploração de profissões, empregos, e organizações realizada nos últimos três meses, (Exploração com locus no Meio), bem como menor grau de exploração pessoal e de retrospeção realizada nos últimos três meses (Exploração com locus em Si Próprio/a) e, menor quantidade de informação adquirida sobre profissões, empregos, organizações e sobre si próprio/a (Quantidade de Informação). Apresentam, igualmente, menos satisfação com a informação obtida sobre as profissões, empregos e organizações mais relacionadas com os seus interesses, capacidades e necessidades (Satisfação com a Informação), e maior quantidade de stress indesejado como função do processo de tomada de decisão, por comparação a outros acontecimentos (Stress na Tomada de Decisão).

Relativamente à indecisão vocacional, constata-se a existência de diferenças estatisticamente significativas entre filhos de pais em condição de emprego e filhos de pais em que um dos pais está na condição de desemprego, no sentido de níveis mais elevados de indecisão vocacional, para os filhos de pais em condição de desemprego.

A Tabela 3 apresenta os resultados das análises de diferenças entre rapazes e raparigas em função da condição de emprego/desemprego dos pais. 
Faria, L. C. (2013). Influência da Condição de Emprego/Desemprego dos Pais na Exploração e Indecisão Vocacional dos Adolescentes.

Tabela 2

Exploração e Indecisão de Carreira: Diferenças em Função da Condição de Emprego/Desemprego dos Pais

\begin{tabular}{|c|c|c|c|c|c|c|c|}
\hline \multirow[t]{2}{*}{ Dimensões de Exploração Vocacional } & & \multicolumn{2}{|c|}{$\begin{array}{c}\text { Pais } \\
\text { Desempregados }\end{array}$} & \multicolumn{2}{|c|}{$\begin{array}{c}\text { Pais } \\
\text { Empregados }\end{array}$} & \multirow[t]{2}{*}{$t$} & \multirow[t]{2}{*}{$p$} \\
\hline & & $M$ & $D P$ & $M$ & $D P$ & & \\
\hline \multirow[t]{5}{*}{ Crenças de Exploração Vocacional } & $\mathrm{EE}$ & 9,413 & 2,271 & 10,043 & 2,319 & 13,374 & $<0,001$ \\
\hline & $\mathrm{CR}$ & 8,205 & 2,908 & 9,411 & 2,807 & 0,044 & 0,835 \\
\hline & IE & 37,226 & 6,449 & 37,545 & 6,769 & 0,638 & 0,425 \\
\hline & II & 14,501 & 3,077 & 14,453 & 2,763 & 3,473 & 0,063 \\
\hline & IPP & 10,760 & 2,463 & 10,985 & 2,540 & 0,779 & 0,378 \\
\hline \multirow[t]{4}{*}{ Processo de Exploração Vocacional } & EM & 10,128 & 3,430 & 12,386 & 3,613 & 14,682 & $<0,001$ \\
\hline & ES & 15,497 & 3,982 & 16,223 & 4,089 & 6,409 & 0,012 \\
\hline & ESI & 4,030 & 1,617 & 4,980 & 1,802 & 1,830 & 0,177 \\
\hline & QI & 7,205 & 2,351 & 9,656 & 2,339 & 35,113 & $<0,001$ \\
\hline \multirow[t]{3}{*}{ Reacções à Exploração Vocacional } & SI & 8,350 & 2,341 & 9,876 & 2,328 & 19,580 & $<0,001$ \\
\hline & SE & 15,313 & 4,643 & 15,365 & 4,392 & 0,020 & 0,968 \\
\hline & STD & 23,947 & 7,101 & 21,766 & 7,424 & 3,811 & 0,050 \\
\hline Indecisão Vocacional & & 35,97 & 6,13 & 33,53 & 7,42 & 13,238 & $<0,001$ \\
\hline
\end{tabular}

Nota. EE- Estatuto de emprego; CR - Certeza dos resultados; IE - Instrumentabilidade externa; II - Instrumentabilidade interna; IPP - Importância da posição preferida; EM - Exploração do meio; ES - Exploração de si próprio; ESI - Exploração sistemática intencional; QI - Quantidade de informação; SI - Satisfação com a informação; SE - Stress na exploração; SD - Stress na tomada de decisão.

Tabela 3

Exploração e Indecisão de Carreira: Diferenças de Sexo em Função da Condição de Emprego/Desemprego dos Pais

\begin{tabular}{|c|c|c|c|c|c|c|c|c|}
\hline & & is Desempregados & & & & is Empregados & & \\
\hline & Raparigas $(n=70)$ & Rapazes $(n=73)$ & & & Raparigas $(n=120)$ & Rapazes $(n=58)$ & & \\
\hline & $M-D P$ & $M-D P$ & $t$ & $p$ & $M-D P$ & $M-D P$ & $t$ & $p$ \\
\hline $\mathrm{EE}$ & $10,21-2,23$ & $9,60-1,99$ & 1,719 & 0,088 & $9,03-2,18$ & $10,15-2,33$ & $-3,057$ & $<0,001$ \\
\hline $\mathrm{CR}$ & $8,66-2,89$ & $8,44-2,95$ & 0,435 & 0,664 & $7,97-2,88$ & $8,66-2,95$ & $-1,493$ & 0,138 \\
\hline IE & $38,64-6,95$ & $36,12-7,80$ & 2,041 & $<0,05$ & $38,19-6,78$ & $37,21-6,01$ & 0,979 & 0,329 \\
\hline II & $15,09-2,69$ & $14,60-2,86$ & 1,073 & 0,285 & $14,61-3,23$ & $14,18-2,90$ & 0,890 & 0,375 \\
\hline IPP & $10,73-2,70$ & $11,03-2,56$ & $-0,696$ & 0,487 & $10,47-2,47$ & $11,43-2,36$ & $-2,513$ & $<0,001$ \\
\hline EM & $8,21-2,28$ & $8,62-2,65$ & $-1,007$ & 0,315 & $7,08-2,38$ & $7,48-2,28$ & $-1,077$ & 0,283 \\
\hline ES & $4,19-1,52$ & $4,39-1,78$ & $-0,738$ & 0,462 & $3,94-1,55$ & $4,17-1,76$ & $-0,842$ & 0,402 \\
\hline ESI & $11,64-3,50$ & $10,62-3,77$ & 1,836 & 0,069 & $10,10-3,45$ & $10,16-3,46$ & $-0,105$ & 0,917 \\
\hline QI & $16,18-4,05$ & $15,41-3,40$ & 1,133 & 0,259 & $15,58-4,04$ & $15,22-4,01$ & 0,558 & 0,578 \\
\hline SI & $9,50-2,42$ & $9,14-2,26$ & 0,916 & 0,361 & $8,11-2,35$ & $8,76-2,32$ & $-1,708$ & 0,089 \\
\hline SE & $14,45-4,61$ & $14,87-4,37$ & $-0,560$ & 0,576 & $15,33-5,07$ & $15,35-5,07$ & $-0,026$ & 0,979 \\
\hline STD & $22,27-7,58$ & $20,90-7,39$ & 1,094 & 0,276 & $24,42-7,10$ & $23,05-7,01$ & 1,218 & 0,225 \\
\hline ID & $33,83-6,13$ & $33,93-7,70$ & $-0,089$ & 0,930 & $36,53-6,55$ & $34,83-5,05$ & 1,748 & 0,058 \\
\hline
\end{tabular}

Nota. EE- Estatuto de emprego; CR - Certeza dos resultados; IE - Instrumentabilidade externa; II - Instrumentabilidade interna; IPP - Importância da posição preferida; EM - Exploração do meio; ES - Exploração de si próprio; ESI - Exploração sistemática intencional; QI - Quantidade de informação; SI - Satisfação com a informação; SE - Stress na exploração; SD - Stress na tomada de decisão; ID - Indecisão Vocacional. 
A análise da Tabela 3 permite verificar a existência de diferenças estatisticamente significativas nos dois grupos (rapazes e raparigas), quer na condição de emprego, quer na condição de desemprego dos pais. Verificam-se diferenças estatisticamente significativas entre rapazes e raparigas na dimensão Instrumentalidade Externa, no sentido de melhores resultados para as raparigas, cujos pais se encontram na condição de desemprego. Ou seja, as raparigas filhas de pais desempregados parecem acreditar mais na probabilidade de a exploração do mundo profissional concorrer para atingir objetivos vocacionais, que os rapazes filhos de pais na mesma condição.

Verificam-se, igualmente, diferenças estatisticamente significativas entre rapazes e raparigas, filhos de pais na condição de emprego, desta feita nas dimensões Estatuto de emprego e Importância da posição preferida, com melhores resultados para os rapazes. $\mathrm{O}$ que parece significar que os rapazes filhos de pais empregados parecem crer que as possibilidades de emprego na área preferida são favoráveis, e atribuir níveis mais elevados de importância à realização da preferência vocacional, que as raparigas, igualmente filhas de pais empregados.

\section{Discussão e Conclusão}

Com base na revisão teórica realizada, é indiscutível que o desenvolvimento vocacional, sendo parte integrante do desenvolvimento humano (Carvalho, 2007; Gonçalves \& Coimbra, 2007), é um processo interativo entre o indivíduo e os múltiplos ambientes nos quais se encontra inserido (e.g, Blustein, 2004; Whiston \& Keller, 2004). Um dos ambientes com maior influência sobre o processo de desenvolvimento vocacional dos adolescentes é, tal como previamente mencionado, o contexto familiar.

Este artigo teve como objetivo fundamental analisar a influência da condição de emprego versus desemprego dos pais na exploração e indecisão vocacional. $\mathrm{Na}$ amostra em estudo foi possível identificar padrões diferenciados de exploração e indecisão vocacional em função da condição de emprego versus desemprego dos pais dos adolescentes, o que chama a atenção, mais uma vez, para a importância de considerar, na intervenção vocacional, as características pessoais e do desenvolvimento vocacional de cada aluno e de grupos específicos de alunos, quando queremos apoiar o seu bem-estar e crescimento pessoal e social.

Tal como teoricamente esperado, de uma maneira geral, os resultados da investigação demonstram que os filhos de pais desempregados tendem a evidenciar um menor grau de exploração vocacional, a par de uma maior tendência para indecisão e dificuldade em comprometer-se com um objetivo educativo ou profissional específico, fruto das crenças negativas e da elevada ansiedade (Neblett \& Cortina, 2006; Silva, 2004).

Os resultados são também consistentes com a teoria e investigação da exploração vocacional que sugerem que a incerteza dos resultados da exploração pode estar na base de níveis elevados de ansiedade situacional associada à exploração e à tomada de decisão vocacional (cf. Blustein \& Phillips, 1988; Faria \& Taveira, 2006; Stumpf et al., 1983). E, poderão estar relacionados, igualmente, com o facto de os filhos de pais desempregados apresentarem menores índices de Estatuto de Emprego, Exploração com locus no Meio, Quantidade de Informação e Satisfação com a informação, dimensões que apresentam associações negativas com a indecisão vocacional (Taveira, 1997). Ou seja, quanto mais baixos forem os valores nas dimensões assinaladas, maior é a probabilidade dos jovens aumentarem o nível de indecisão vocacional.

De facto, os filhos de pais desempregados, por um lado, sendo menos otimistas, possuindo uma orientação negativa e, apresentando maiores níveis de stress, terão mais dificuldades em tomar decisões, reflectindo-se nos seus comportamentos de exploração e de tomada de decisão (Neblett \& Cortina, 2006; Sobral et al., 2009). Por sua vez, os filhos de pais empregados apresentam expectativas de sucesso mais elevadas (Galambos \& Silbereisen, 1987), podendo ter uma visão mais otimista do seu próprio futuro profissional e sentindo-se mais motivados relativamente às suas escolhas futuras (Sobral et al., 2009). De acordo com a teoria da exploração vocacional, este tipo de sentimentos de confiança no futuro constitui um factor cognitivo-motivacional da exploração vocacional futura (Stumpf et al., 1983), que, por sua vez, favorece a activação do processo de exploração vocacional (Taveira, 1997). Por exemplo, os jovens que aspiram e consideram ser possível aceder a uma posição favorável no mercado de trabalho no futuro, são aqueles que se envolvem mais, e de modo mais sistemático, na exploração vocacional (e.g., Sugalski \& Greenhaus, 1986). Existe, também, uma tendência para os filhos de pais empregados apresentarem mais e maior satisfação com a informação obtida sobre as profissões, empregos e organizações mais relacionadas com os seus interesses, capacidades e necessidades, e menor stress em função do processo de tomada de decisão.

Assim sendo, estes dados vão de encontro à teoria e investigação da auto-eficácia vocacional (e.g., Brown \& Lent, 1996; Lent \& Brown, 1996), que sugere que a confiança face às tarefas de exploração vocacional pode facilitar a tomada de decisão. Os filhos de pais empregados apresentam, igualmente, maior grau de exploração pessoal e de retrospecção e de exploração de profissões, empregos, e organizações realizada nos últimos três meses. Krumboltz (1996) refere a este propósito que a exploração vocacional na adolescência e vida adulta pode ser facilitada pelos sistemas de reforço e pela aprendizagem vicariante (e.g., Corrigan, Dell, Lewis, \& Schmidt, 1980; Heppner \& Claiborn, 1989; Heppner \& Dixon, 1981). Diferentes formas de reforço ou de modelagem social provocam aumentos na quantidade e na frequência de comportamentos de procura e informação (e.g., Krumboltz \& Schoeder, 1965). Assim, se os adolescentes virem os seus pais envolvidos e satisfeitos com o seu trabalho, tendem a apresentar um nível de bem-estar mais elevado, desenvolvendo atitudes positivas face ao trabalho (Barling, Dupre, \& Hepburn, 1998). 
Faria, L. C. (2013). Influência da Condição de Emprego/Desemprego dos Pais na Exploração e Indecisão Vocacional dos Adolescentes.

Ainda neste ponto gostaríamos de referir que os resultados não significativos do ponto de vista estatístico das escalas de Instrumentalidade Interna, Instrumentalidade Externa e Importância de obter uma Posição Preferida, poderão estar relacionados com o carácter estável e cristalizado que este tipo de crenças pode assumir, resultado da história de aprendizagem, questões de género e culturais (Krumboltz, 1996).

Os resultados relativos às diferenças de sexo chamam a atenção, mais uma vez, para a importância de considerar as questões de género em relação estreita com as abordagens à orientação e desenvolvimento vocacional de jovens. Neste sentido, é fundamental que a orientação vocacional se estenda aos representantes parentais de cada jovem, no sentido de haver uma escolha vocacional mais ajustada a cada situação e desprovida de mecanismos estereotipados que condicionam o futuro de muitas raparigas (cf. Saavedra, 2005; Taveira \& Nogueira, 2004).

Em traços gerais, a principal conclusão que se retira, à semelhança de outras investigações na área (Gonçalves, 2008; Pinto \& Soares, 2001, 2002; Young et al., 2006), é de que a família é um contexto de vida decisivo e primordial de aquisição, promoção e desenvolvimento de competências de vida, podendo ser facilitadora ou inibidora do desenvolvimento global do indivíduo e, consequentemente, do desenvolvimento vocacional. Neste sentido, salienta-se a necessidade dos profissionais de orientação desenvolverem intervenções que, desde logo, visem o envolvimento dos pais (e.g., Carvalho, 2007; Gonçalves \& Coimbra, 2007), e que contribuam para a integração dos esforços de pais e filhos (e.g., Chope, 2006; Gonçalves, 2008). É importante expandir o atual número de intervenções vocacionais que visam apoiar os pais a tornarem-se mais envolvidos nas escolhas de carreira dos seus filhos. Por um lado, a presença e participação dos pais nos programas de intervenção vocacional podem funcionar como um estimulante para a sua eficácia. Por outro lado, essa mesma colaboração parece ter capacidade para incentivar os pais a serem mais apoiantes e participativos no desenvolvimento dos seus filhos adolescentes (e.g., Downing \& D'Andrea, 1997). Além disso, assume particular importância na intervenção vocacional o apoio ao adolescente na compreensão da influência que a família exerce sobre as suas escolhas vocacionais (e.g., Chope, 2006; Diemer, 2007). Na maior parte dos casos, os adolescentes que pedem apoio para uma tomada de decisão iminente não estão conscientes acerca do modo como a sua família tem implicações no seu desenvolvimento vocacional. Deverá ser da responsabilidade do psicólogo apoiar o cliente no levantamento da sua história familiar, dos valores, crenças, mitos, bem como no reconhecimento do seu impacto ao nível da tomada de decisão vocacional. O jovem não pode ser entendido como uma vítima da influência do sistema familiar (Chope, 2006; Diemer, 2007), na medida em que ele é parte integrante desse mesmo sistema. Neste sentido, poderá ser particularmente vantajoso que o psicólogo vocacional auxilie o adolescente não só a desenvolver consciência acerca dos múltiplos fatores que influenciam as suas escolhas, mas também a assumir a responsabilidade pelas mesmas.

Para além das questões que nos parecem especialmente relevantes para a prática da orientação vocacional, pensamos que é importante referir algumas das limitações desta investigação. Em primeiro lugar, precisamos tecer algumas considerações em termos das variáveis que podem interferir nestes resultados. Há muitas outras variáveis intervenientes, por exemplo, o fato de os alunos serem estudantes de escolas públicas da zona rural, onde talvez as perspetivas de emprego e possibilidades de exploração e escolha já sejam restritas. $\mathrm{O}$ fato de se tratar de uma amostra de conveniência também constitui uma limitação à generalização dos resultados obtidos. Outra dificuldade com que nos deparamos prende-se com a bateria de instrumentos utilizada. Apesar dos instrumentos estarem adaptados à população portuguesa (Taveira, 1997), pelo facto de serem de auto relato, estão sujeitos a erros de interpretação e a fatores de desejabilidade social.

Investigações futuras poderiam averiguar, além das variáveis intervenientes referidas, o impacto do estatuto socioeconómico, bem como das relações de estabilidade e/ou precariedade dos vínculos. Uma outra sugestão para estudos posteriores é, também, centrarmo-nos no impacto das relações pais-filhos na transmissão intergeracional do significado do trabalho e da parentalidade (Sobral et al., 2009). Em suma, a realização de mais estudos sobre a influência parental no desenvolvimento vocacional continua a ser muito necessária. Esperamos que este trabalho tenha contribuído para encorajar mais esforços multidisciplinares no estudo e intervenção vocacional junto de adolescentes.

\section{Referências}

Alarcão, M., \& Gaspar, M. F. (2007). Imprevisibilidade familiar e suas implicações no desenvolvimento individual e familiar. Paidéia (Ribeirão Preto), 17(36), 89-102.

Amundson, N. E., \& Penner, K. (1998). Parent involved career exploration. The Career Development Quarterly, 47, 135-144.

Bacon, C., \& Lerner, R. (1975). Effects of maternal employment status on the development of vocational-role perception in females. The Journal of Genetic Psychology, 126, 187-194.

Bardagi, M. P., \& Hutz, C. S. (2008). Apoio parental percebido no contexto da escolha inicial e da evasão de curso universitário. Revista Brasileira de Orientação Profissional, 9(2), 31-44.

Barling, J., Dupre, K. E., \& Hepburn, C. G. (1998). Effects of parent's job insecurity on children's work beliefs and attitudes. Journal of Applied Psychology, 83, 112-118.

Berríos-Allison, A. C. (2005). Family influences on college students' occupational identity. Journal of Career Assessment, 13(2), 233-247.

Blustein, D. L. (2004). Moving from the inside out: Further explorations of the family of origin/career development linkage. The Counseling Psychologist, 32(4), 603-611.

Blustein, D. L., \& Phillips, S. (1988). Individual and contextual factors in career exploration. Journal of Vocational Behavior, 33, 203-216.

Boing, E., Crepaldi, M. A., \& More, C. L. (2008). Pesquisa com famílias: Aspectos teórico-metodológicos. Paidéia (Ribeirão Preto), 18(40), 251-266. 
Brown, S. D., \& Lent, R. W. (1996). Applying social cognitive theory to career counselling: An introduction. Career Development Quarterly, 44, 354-366.

Carvalho, M. (2007). Perspectivas sobre a influência parental na execução de planos de carreira no ensino secundário (Dissertação de mestrado, Universidade do Minho, Braga, Portugal).

Carvalho, M., \& Taveira, M. C. (2009). Influência de pais nas escolhas de carreira dos filhos: Visão de diferentes atores. Revista Brasileira de Orientação Profissional, 10(2), 33-41.

Carvalho, M., \& Taveira, M. C. (2010). O papel dos pais na execução de planos de carreira no Ensino Secundário: Perspectivas de pais e de estudantes. Análise Psicológica, 2(28), 333-341.

Castellino, D., Lerner, J., Lerner, R., \& Eye, A. (1999). Maternal employment and education: Predictors of young adolescent career trajectories. Applied Developmental Science, 2, 114-126.

Chope, R. C. (2006). Family matters: The influence of the family in career decision making. Austin, TX: Pro-Ed.

Cinamon, R. (2002). Father's unemployment and career related variables of his adolescent child. International Journal for the Advancement of Counselling, 23, 292-309.

Corrigan, J. D., Dell, D. M., Lewis, K. N., \& Schmidt, L. D. (1980). Counseling as a social influence process: A review. Journal of Counseling Psychology, 27(4), 395-441.

Creamer, E. G., \& Laughlin, A. (2005). Self-authorship and women's career decision making. Journal of College Student Development, 46(1), 13-27.

Crouter, A., \& Bumpus, M. (2001). Linking parent's work stress to children's and adolescent's psychological adjustment. Current Directions in Psychological Science, 10, 156-159.

Diemer, M. (2007). Parental and school influences upon the career development of poor youth of color. Journal of Vocational Behavior, 70(3), 502-524.

Downing, J., \& D'Andrea, L. M. (1997). An effective career development program for parents. Journal of Employment Counseling, 34, 55-64.

Faria, L., \& Taveira, M. C. (2006). Avaliação dos Resultados da Intervenção Psicológica Vocacional: Um estudo de avaliação de resultados em finalistas do $3^{\circ}$ ciclo do ensino básico. In N. Santos, M. L. Lima, M. Melo, A. Candeias, M. L. Grácio, \& A. Calado (Eds.), Actas do VI Simpósio Nacional de Investigação Bibliografia em Psicologia (pp. 24-48). Évora, Portugal: Departamento de Psicologia, Universidade de Évora.

Galambos, N., \& Silbereisen, R. (1987). Income change, parental life outlook, and adolescent expectation of job success. Journal of Marriage and the Family, 49, 141-149.

Gonçalves, C. M. (2006). A família e a construção de projectos vocacionais de adolescentes e adolescentes (Tese de doutorado, Faculdade de Psicologia e Ciências da Educação, Universidade do Porto, Portugal).

Gonçalves, C. M. (2008). Pais aflitos, filhos com futuro incerto? Um estudo sobre a influência das famílias na orientação vocacional dos jovens. Lisboa, Portugal: Edições Fundação Calouste Gulbenkian.

Gonçalves, C. M., \& Coimbra, J. L. (2007). O papel dos pais na construção de trajectórias vocacionais dos seus filhos. Revista Brasileira de Orientação Profissional, 8(1), 1-17.

Hanisch, K. (1999). Job loss and unemployment research from 1994 to 1998: A review and recommendations for research and intervention. Journal of Vocational Behavior, 55, 188-220.

Hartung, P. J., Porfeli, E. J., \& Vondracek, F. W. (2005). Child vocational development: A review and reconsideration. Journal of Vocational Behavior, 66, 385-419.
Heppner, P. P., \& Claiborn, C. D. (1989). Social influence research in counseling: A review and critique. Journal of Counseling Psychology, 36(3), 365-387.

Heppner, P. P., \& Dixon, D. (1981). A review of the interpersonal influence process n counseling. Personnel and Guidance Journal, 59, 542-550.

Ketterson, T. U., \& Blustein, D. L. (1997). Attachment relationships and the career exploration process. The Career Development Quarterly, 46, 167-178.

Kracke, B. (1997). Parental behaviors and adolescents' career exploration. The Career Development Quarterly, 45, 341-350.

Krumboltz, J. D. (1996). A learning theory of career counseling. In M. L. Savickas \& W. B. Walsh (Eds.), Handbook of Career Counseling Theory and Practice (pp. 55-80). Palo Alto, CA: Consulting Psychologists Press.

Krumboltz, J. D., \& Schoeder, W. W. (1965). Promoting career planning through reinforcement. Personnel and Guidance Journal, 44, 19-26.

Lent, R. W., \& Brown, S. D. (1996). Social cognitive approach to career development: An overview. The Career Development Quarterly, 44, 310-321.

Leon, L. M., \& Iguti, A. M. (2003). Saúde em tempos de desemprego. In L. A. M. Guimarães \& S. Grubits (Eds.), Série Saúde Mental e Trabalho (pp. 196-210). São Paulo, SP: Casa do Psicólogo.

Lindstrom, L., Doren, B., Metheny, J., Johnson, P., \& Zane, C. (2007). Transition to employment: Role of the family in career development. Exceptional Children, 73(3), 348-366.

Murray, C. (2009). Parent and teacher relationships as predictors of school engagement and functioning among low-income urban youth. Journal of Early Adolescence, 29, 376-404.

Neblett, N., \& Cortina, K. (2006). Adolescent's thoughts about parent's jobs and their importance for adolescent's future orientation. Journal of Adolescence, 29, 795-811.

Osipow, S. H., Carney, C. G., Winer, J. L., Yanico, B., \& Koshier, M. (1976). The Career Decision Scale ( $3^{\text {rd }}$ Rev. ed.). Columbus, OH: Marathon Consulting \& Press.

Otto, L. B. (2000). Youth perspectives on parental career influence. Journal of Career Development, 27(2), 111-118.

Pinto, H. R., \& Soares, M. C. (2001). Influência parental no desenvolvimento vocacional (Relatório de investigação). Lisboa, Portugal: Instituto de Orientação Profissional.

Pinto, H. R., \& Soares, M. C. (2002). Influência parental na carreira: Evolução de perspectivas na teoria, na investigação e na prática. Psychologica, 26, 135-149.

Pratta, E. M., \& Santos, M. A. (2007). Opiniões dos adolescentes do ensino médio sobre o relacionamento familiar e seus planos para o futuro. Paidéia (Ribeirão Preto), 17(36), 103-114.

Saavedra, L. (2005). Aprender a ser rapariga, aprender a ser rapaz: Teorias e práticas da escola. Coimbra, Portugal: Almedina.

Schliebner, C., \& Peregoy, J. (1994). Unemployment effects on the family and the child: Interventions for counsellors. Journal of Counselling and Development, 72, 368-372.

Silva, J. T. (2004). A eficácia da intervenção vocacional em análise: Implicações para a prática psicológica. In M. C. Taveira (Ed.), Desenvolvimento vocacional ao longo da vida. Fundamentos, princípios e orientações (pp. 95-125). Coimbra, Portugal: Almedina.

Sobral, J. M., Gonçalves, C. M., \& Coimbra, J. L. (2009). A influência da situação profissional parental no desenvolvimento vocacional dos adolescentes. Revista Brasileira de Orientação Profissional, 10(1), 11-22. 
Faria, L. C. (2013). Influência da Condição de Emprego/Desemprego dos Pais na Exploração e Indecisão Vocacional dos Adolescentes.

Stumpf, S. A., Colarelli, M. S., \& Hartman, K. (1983). Development of the career exploration survey (CES). Journal of Vocational Behavior, 22, 191-226.

Sugalski, T. D., \& Greenhaus, J. H. (1986). Career exploration and goal setting among managerial employees. Journal of Vocational Behavior, 29, 102-114.

Taveira, M. C. (1997). Exploração e desenvolvimento vocacional de adolescentes. Estudo sobre as relações entre a exploração, a identidade e a indecisão vocacional (Tese de doutorado, Universidade do Minho, Braga, Portugal).

Taveira, M. C., \& Nogueira, C. (2004). Estudos de género e psicologia vocacional: Confronto de teorias e implicações para a intervenção vocacional. In M. C. Taveira (Coord.), Desenvolvimento vocacional ao longo da vida. Fundamentos, princípios e orientações (pp. 57-82). Coimbra, Portugal: Almedina.

Turner, S., \& Lapan, R. T. (2002). Career self-efficacy and perceptions of parent support in adolescent career development. The Career Development Quarterly, 51, 44-55.

Vasconcelos, Z. B., \& Oliveira, I. D. (Eds.). (2004). Orientação vocacional: Alguns aspectos teóricos, técnicos e práticos. São Paulo, SP: Vetor.

Vignolli, E., Croity-Belz, S., Chapeland, V., Fillipis A., \& Garcia, M. (2005). Career exploration in adolescents: The role of anxiety, attachment, and parenting style. Journal of Vocational Behavior, 67, 153-168.

Whiston, S. C., \& Keller, B. K. (2004). The influences of the family of origin on career development: A review and analysis. The Counseling Psychologist, 32(4), 493-568.

Wolfe, J., \& Betz, N. (2004). The relationship of attachment variables to career decision-making self-efficacy and fear of commitment. The Career Development Quarterly, 54(4), 363-369.

Young, R. A., Marshall, S. K., Domene, J. F., Arato-Bollivar, J., Hayoun, R. Marsahll, E. G, ...Valach L. (2006). Relationships, communication, and career in the parent-adolescent projects of families with and without challenges. Journal of Vocational Behavior, 68, 1-23. 